\title{
Synthesis and Antimicrobial Activity of 2-[4- (Substituted Benzylidenamino)-5-(Substituted Phenoxymethyl)-4H-1,2,4-Triazol-3-yl thio] Acetic Acid Derivatives
}

\author{
R. D. HUNASHAL ${ }^{1, *}$, D. SATYANARAYANA ${ }^{2}$ AND V. S. MADDI ${ }^{3}$ \\ 1* Department of Pharmacology, Karnataka Institute of Medical Sciences, Hubli-580 021, \\ Karnataka, India , e-mail:rajeshrd3@rediffmail.com \\ ${ }^{2}$ Department of Pharmaceutical Chemistry, NGSM Institute of Pharmaceutical Sciences, \\ Paneer, Deralakatte, Mangalore-574 160, Karnataka. India \\ ${ }^{3}$ Department of Pharmaceutical Chemistry, K.L.E S's College of Pharmacy, Hubli-580 031, \\ Karnataka, India
}

Received 08 November 2011; Accepted 15 January 2012

\begin{abstract}
: 2-[4-(substituted benzylidenamino)-5-(substituted phenoxymethyl)$4 H-1,2,4-t r i a z o l-3-y l$ thio] acetic acid (3a-j) derivatives have been synthesized by the reaction between 4-(substituted benzylidenamino)-5-(substituted phenoxymethyl)-2H-1,2,4-triazol-3(4H)-thiones (2a-j) and chloroacetic acid in presence of catalytic amount of pyridine. All these compounds were screened for antimicrobial activity. The compounds $\mathbf{3 h}$ and $\mathbf{3} \mathbf{j}$ exhibited good antifungal activity against $A$. niger, $C$. neoformans, and A. fumigatus at MIC of 0.25 $\mu \mathrm{g} / \mathrm{ml}$ compared to standard drug fluconazole with MIC of $1 \mu \mathrm{g} / \mathrm{mL}$.
\end{abstract}

Keywords: 1,2,4-Triazole, Schiff Base, 1,2,4-Triazolyl thioacetic acid, Antibacterial activity, Antifungal activity.

\section{Introduction}

Fungal infection became an important complication and a major cause of morbidity and mortality in immuno compromised individuals such as those suffering from tuberculosis, cancer, AIDS, and also in organ transplant cases. ${ }^{1}$ The triazolyl ring seems to play a key role as antifungal drugs, for example, fluconazole, voriconazole, and itraconazole, which are strong inhibitors of lanosterol $14 \alpha$-demethylase and orally active have been widely used in antifungal chemotherapy. In recent years the developments of resistance to currently available antifungal azoles as well as clinical failures in the treatment of fungal infections have been reported. ${ }^{2-5}$ Thus; much effort to develop novel antifungal agents which are more safe and efficacious is still being made. Many compounds bearing the 1,2,4-triazole nucleus 
have reported to possess wide variety biological activities viz. anticonvulsant ${ }^{6,7}$ antibacterial $^{8}$, antifungal ${ }^{9}$, antitubercular ${ }^{10}$, anticancer ${ }^{11}$, and anti-inflammatory. ${ }^{12}$

In view of these facts and in continuation of our research program on synthesis and biological importance of various heterocyclic Schiff's bases, ${ }^{13,14}$ now we are reporting the synthesis and antimicrobial activity of substituted 1,2,4-triazole (3a-j) derivatives as shown in scheme-1.<smiles>[R]c1cccc(OCc2n[nH]c(=S)n2N)c1</smiles><smiles>[R][R]1cccc(/C=N/n2c(COc3cccc([R])c3)nnc2SCC(=O)O)c1</smiles>

3a-j<smiles>[R]c1cccc(OCc2nnc(S)n2N)c1</smiles>

1

where $\mathrm{R}=\mathrm{H}(1 \mathrm{a}), 2,4-\mathrm{Cl}(1 \mathrm{~b})$<smiles></smiles><smiles>[R]C[C@H](C)[C@H](C)O</smiles>

$\overleftarrow{\mathrm{ClCH}_{2} \mathrm{COOH}}$

$2 a-j$

\section{Scheme 1}

\section{Experimental}

All chemicals were purchased from Acros organics, Sigma-Aldrich, Lancaster Co. Thin layer chromatography using (TLC) pre-coated silica gel plates from E. Merck and Co.. Melting points of synthesized compounds were determined in Thermonik melting point apparatus and are uncorrected. The IR spectra were recorded on Thermo Nicolet FTIR-200 Spectrometer, The ${ }^{1} \mathrm{H}$ NMR recorded on Bruker AVANCE II 400 (in $\mathrm{CDCl}_{3} / \mathrm{DMSO}_{\mathrm{d}}$ as solvent. Chemical shifts are reported in $\delta$ ppm units with respect to TMS.

\section{General Procedure}

Synthesis of 4-(substituted benzylidenamino)-5-(substituted phenoxymethyl)-2H-1,2,4triazole-3(4H)-thione (2a-j)

A mixture of N'-4-amino-5-(substituted phenoxymethyl)-2H-1,2,4-triazole-3(4H)-thione (1) $(0.01 \mathrm{~mol})$ and the corresponding various aromatic aldehydes $(0.01 \mathrm{~mol})$ in ethanol $(25 \mathrm{~mL})$ was treated with concentrated $\mathrm{HCl}(0.5 \mathrm{~mL})$ and refluxed for $2 \mathrm{hr}$. The reaction mixture was 
cooled to room temperature and the separated crystalline compound is filtered and recrystallized from ethanol to give $\mathbf{2} \mathbf{a}-\mathbf{j}$.

Synthesis of 2-[4-(substituted benzylidenamino)-5-(substituted phenoxymethyl)-4H-1,2,4triazol-3-yl thio] acetic acid (3a-j)

Equimolar proportions of $\mathbf{2 a - j}$ and chloroacetic acid were dissolved in ethanol containing 34 drops of pyridine and refluxed for 2-3 hrs, on pouring the reaction mixture into cold water a solid 3a-j was separated which was filtered, washed with water, and recrystallized from ethanol to give $\mathbf{3 a - j}$.

\section{Antimicrobial Screening}

Antimicrobial activity of novel compounds screened against Streptococcus aureus (ATCC 9144, Sa), Bacillus subtilis (ATCC 6633, Bs). Psedomonas aeruginosa (ATCC 25668,Pa), Escherichia coli (ATCC 25922, Ec). Candida albicans (ATCC 2091Ca), Cryptococcus neoformans (108), Aspergillus niger (An), Aspergillus fumigatus (Af), and MIC was determined agar-dilution method. ${ }^{15,16}$

\section{Results and Discussion}

Synthesis

The IR spectrum of compound 3a-j showed a absorption bands at range of 3281 to 3091 $\mathrm{cm}^{-1}$ was accounting for $\mathrm{OH}$ and 1735 to $1715 \mathrm{~cm}^{-1}$ indicating the presence $\mathrm{C}=\mathrm{O}$ of $\mathrm{COOH}$ group. In the ${ }^{1} \mathrm{H}$ NMR compounds displayed a broad singlet at $\delta 14.0$ to 13.5 was attributed of $\mathrm{COOH}$ and singlet at $\delta 2.8$ to 2.1 was ascribed to $\mathrm{S}^{-\mathrm{CH}_{2}}$. It suggests the formation of S$\mathrm{CH}_{2}-\mathrm{COOH}$ at $5^{\text {th }}$ position of triazole. Further the ${ }^{13} \mathrm{C}$ NMR data displayed characteristic signal at $\delta 39.4$ to 29.5 due to $\mathrm{S}_{-} \mathrm{CH}_{2}$. It indicates the formation of thioacetic acid by the reaction of $\mathrm{SH}$ with chloroacetic acid. The structure of compounds was further supported by mass spectral data. The physical and analytical data of all compounds are tabulated in Table 1 and Table 2.

\section{Antimicrobial Activity}

Antifungal and antibacterial screening data revealed that all the compounds showed moderate to good antifungal and weak antibacterial activity when compared with standard drugs respectively. Antibacterial and antifungal results are given in Table 3.

The antifungal activity, revealed that the compound $\mathbf{3 h}$ substituted at m- methoxy and at p-hydroxy groups, the compounds $\mathbf{3} \mathbf{j}$ substituted at position of p-hydroxy group on imine phenyl ring showed grater activity respectively and compound $\mathbf{3 f}, \mathbf{3 g}, \mathbf{3 i}$ showed equipotent activity against A. niger, $C$. neoformans, and A. fumigatus compared to standard drug fluconazole. Compound $\mathbf{3 g}$ with nitro group on m-position showed greater activity against C. albicans and equipotent with other fungal strains. This indicates that, the electron withdrawing substituent's on imine phenyl ring showed more potent activity than standard drug, where as compound $\mathbf{3 f}, \mathbf{3 g}, \mathbf{3 h}, \mathbf{3 i}, \mathbf{3} \mathbf{j}$ with two chloro substituted on o-and p-position on phenoxymethyl ring showed more activity compared to no substitution phenoxymethyl ring in the series. Hence, it reveals that the compounds having electron withdrawing substituent's on imine phenyl ring were found to be more active. 
Table 1. Spectral data of synthesised compounds 3a-j.

\begin{tabular}{|c|c|c|c|c|}
\hline \multirow[t]{2}{*}{ Entry } & \multirow[t]{2}{*}{$\mathbf{R}$} & \multirow[t]{2}{*}{$\mathbf{R}_{1}$} & \multicolumn{2}{|c|}{ Spectral data } \\
\hline & & & $\mathrm{IR}\left(\mathrm{KBr}, \mathrm{cm}^{-1}\right)$ & $\begin{array}{l}{ }^{1} \mathrm{H} \text { NMR } \\
\left(400 \mathrm{MHz}, \mathrm{DMSO}-\mathrm{d}_{6}, \delta \mathrm{ppm}\right)\end{array}$ \\
\hline $2 \mathrm{~h}$ & $2,4-\mathrm{Cl}$ & $\begin{array}{l}3-\mathrm{OCH}_{3}, \\
4-\mathrm{OH}\end{array}$ & $\begin{array}{l}3200.0(\mathrm{OH}), 3100.2(\mathrm{Ar} \mathrm{C}- \\
\mathrm{H}), 2912.0\left(\mathrm{OCH}_{2}\right), 2360.0 \\
(\mathrm{SH}), 1592.0\left(\mathrm{HC}^{2}=\mathrm{N}\right) \\
1508.5(\mathrm{C}=\mathrm{N}), 810.8(\mathrm{C}-\mathrm{Cl})\end{array}$ & $\begin{array}{l}13.8(\mathrm{~s}, 1 \mathrm{H}, \mathrm{SH}), 9.8(\mathrm{~s}, 1 \mathrm{H} \\
\mathrm{HC}=\mathrm{N}), 7.6-6.9(\mathrm{~m}, 6 \mathrm{H}, \mathrm{Ar}-\mathrm{H}) \\
5.2\left(\mathrm{~d}, 2 \mathrm{H}, \mathrm{OCH}_{2}\right), 3.9(\mathrm{~s}, 1 \mathrm{H}, \\
\mathrm{OH}), 3.8\left(\mathrm{~s}, 3 \mathrm{H}, \mathrm{OCH}_{3}\right)\end{array}$ \\
\hline $2 \mathbf{j}$ & $2,4-\mathrm{Cl}$ & $4-\mathrm{OH}$ & $\begin{array}{l}3044.0(\mathrm{OH}), 2919.5\left(\mathrm{OCH}_{2}\right), \\
2361.7(\mathrm{SH}), 1585.4 \\
(\mathrm{HC}=\mathrm{N}), 1482.8(\mathrm{C}=\mathrm{N}), \\
798.8(\mathrm{C}-\mathrm{Cl}) .\end{array}$ & $\begin{array}{l}13.9(\mathrm{br}, 1 \mathrm{H}, \mathrm{SH}), 10.4(\mathrm{~s}, 1 \mathrm{H}, \\
\mathrm{HC}=\mathrm{N}), 9.8(\mathrm{~s}, 1 \mathrm{H}, \mathrm{OH}), 7.7-6.8 \\
(\mathrm{~m}, 7 \mathrm{H}, \mathrm{Ar}-\mathrm{H}), 5.2(\mathrm{~s}, 2 \mathrm{H}, \\
\left.\mathrm{OCH}_{2}\right) .\end{array}$ \\
\hline $3 \mathbf{a}$ & $\mathrm{H}$ & $\mathrm{H}$ & $\begin{array}{l}3200.2(\mathrm{OH}), 3052.9(\mathrm{Ar} \mathrm{C}- \\
\mathrm{H}), 2918.1\left(\mathrm{OCH}_{2}\right), 1734.0 \\
(\mathrm{C}=\mathrm{O}), 1590.9(\mathrm{C}=\mathrm{N})\end{array}$ & $\begin{array}{l}13.8(\mathrm{~s}, 1 \mathrm{H}, \mathrm{OH}), 10.3(\mathrm{~s}, 1 \mathrm{H} \\
\mathrm{N}=\mathrm{CH}), 7.7-6.9(\mathrm{~m}, 9 \mathrm{H}, \mathrm{Ar}-\mathrm{H}) \\
5.2\left(\mathrm{~s}, 2 \mathrm{H}, \mathrm{OCH}_{2}\right), 2.5(\mathrm{br}, 2 \mathrm{H} \\
\left.\mathrm{CH}_{2}\right)\end{array}$ \\
\hline $3 \mathbf{b}$ & $\mathrm{H}$ & $3-\mathrm{NO}_{2}$ & $\begin{array}{l}3044.0(\mathrm{OH}), 2919.5\left(\mathrm{OCH}_{2}\right), \\
2361.7(\mathrm{SH}), 1585.4 \\
(\mathrm{HC}=\mathrm{N}), 1482.8(\mathrm{C}=\mathrm{N}), \\
798.8(\mathrm{C}-\mathrm{Cl}) .\end{array}$ & $\begin{array}{l}13.9(\mathrm{br}, 1 \mathrm{H}, \mathrm{SH}), 10.4(\mathrm{~s}, 1 \mathrm{H}, \\
\mathrm{HC}=\mathrm{N}), 9.8(\mathrm{~s}, 1 \mathrm{H}, \mathrm{OH}), 7.7-6.8 \\
(\mathrm{~m}, 7 \mathrm{H}, \mathrm{Ar}-\mathrm{H}), 5.2(\mathrm{~s}, 2 \mathrm{H}, \\
\left.\mathrm{OCH}_{2}\right) .\end{array}$ \\
\hline $3 c$ & $\mathrm{H}$ & $\begin{array}{l}3-\mathrm{OCH}_{3}, \\
4-\mathrm{OH}\end{array}$ & $\begin{array}{l}3200.2(\mathrm{OH}), 3048.1(\mathrm{Ar} \mathrm{C}- \\
\mathrm{H}), 2914.2\left(\mathrm{OCH}_{2}\right), 2747.1 \\
\left(\mathrm{CH}_{2}\right), 1734.0(\mathrm{C}=\mathrm{O}), 1587.8 \\
(\mathrm{C}=\mathrm{N})\end{array}$ & $\begin{array}{l}13.7(\mathrm{~s}, 1 \mathrm{H}, \mathrm{OH}), 10.7(\mathrm{~s}, 1 \mathrm{H}, \\
\mathrm{N}=\mathrm{CH}), 8.4-6.8(\mathrm{~m}, 9 \mathrm{H}, \mathrm{Ar}-\mathrm{H}) \\
5.1\left(\mathrm{~s}, 2 \mathrm{H}, \mathrm{OCH}_{2}\right), 2.5(\mathrm{~s}, 2 \mathrm{H}, \\
\left.\mathrm{CH}_{2}\right)\end{array}$ \\
\hline 3d & $\mathrm{H}$ & $\begin{array}{l}4- \\
\mathrm{N}\left(\mathrm{CH}_{3}\right)_{2}\end{array}$ & $\begin{array}{l}3092.3(\mathrm{OH}), 3000.2(\mathrm{Ar} \mathrm{C}- \\
\mathrm{H}), 2911.6\left(\mathrm{OCH}_{2}\right), 2374.0 \\
\left(\mathrm{CH}_{2}\right), 1716.7(\mathrm{C}=\mathrm{O}), 1585.9 \\
(\mathrm{C}=\mathrm{N})\end{array}$ & $\begin{array}{l}13.6(\mathrm{~s}, 1 \mathrm{H}, \mathrm{OH}), 9.7(\mathrm{~s}, 1 \mathrm{H}, \\
\mathrm{N}=\mathrm{CH}), 7.6-6.6(\mathrm{~m}, 9 \mathrm{H}, \mathrm{Ar}-\mathrm{H}) \\
5.1\left(\mathrm{~d}, 2 \mathrm{H}, \mathrm{OCH}_{2}\right), 3.1(\mathrm{~s}, 6 \mathrm{H}, \\
\left.\mathrm{N}-\left(\mathrm{CH}_{3}\right)_{2}\right), 2.1\left(\mathrm{~s}, 2 \mathrm{H}, \mathrm{CH}_{2}\right)\end{array}$ \\
\hline $3 e$ & $\mathrm{H}$ & $4-\mathrm{OH}$ & $\begin{array}{l}3201.1(\mathrm{OH}), 3051.0(\mathrm{Ar} \mathrm{C}- \\
\mathrm{H}), 2916.4\left(\mathrm{OCH}_{2}\right), 1730.0 \\
(\mathrm{C}=\mathrm{O}), 1602.5(\mathrm{C}=\mathrm{N})\end{array}$ & $\begin{array}{l}13.8(\mathrm{~s}, 1 \mathrm{H}, \mathrm{OH} \text { of carboxylic } \\
\text { acid), } 13.5(\mathrm{br}, 1 \mathrm{H}, \mathrm{OH} \text { of } \\
\text { benzylidene }), 9.8(\mathrm{~s}, 1 \mathrm{H}, \\
\mathrm{N}=\mathrm{CH}), 7.7-6.7(\mathrm{~m}, 9 \mathrm{H}, \mathrm{Ar}-\mathrm{H}), \\
5.2\left(\mathrm{~s}, 2 \mathrm{H}, \mathrm{OCH}_{2}\right), 2.5(\mathrm{br}, 2 \mathrm{H},- \\
\left.\mathrm{CH}_{2}\right)\end{array}$ \\
\hline $3 f$ & $2,4-\mathrm{Cl}$ & $\mathrm{H}$ & $\begin{array}{l}3100.2(\mathrm{OH}), 3056.2(\mathrm{Ar} \mathrm{C}- \\
\mathrm{H}), 2920.4\left(\mathrm{OCH}_{2}\right), 2360.9 \\
\left(\mathrm{CH}_{2}\right), 1711.5(\mathrm{C}=\mathrm{O}), \\
1591.4(\mathrm{C}=\mathrm{N}), 752.7(\mathrm{C}-\mathrm{Cl})\end{array}$ & $\begin{array}{l}12.7(\mathrm{br}, 1 \mathrm{H}, \mathrm{SH}), 11.2(\mathrm{~s}, 1 \mathrm{H}, \\
\mathrm{OH}), 9.2(\mathrm{~s}, 1 \mathrm{H}, \mathrm{N}=\mathrm{CH}), 7.2-6.9 \\
(\mathrm{~m}, 8 \mathrm{H}, \mathrm{Ar}-\mathrm{H}), 5.2(\mathrm{~s}, 2 \mathrm{H}, \\
\left.\mathrm{OCH}_{2}\right), 2.8\left(\mathrm{br}, 2 \mathrm{H}, \mathrm{CH}_{2}\right)\end{array}$ \\
\hline $3 g$ & $2,4-\mathrm{Cl}$ & $3-\mathrm{NO}_{2}$ & $\begin{array}{l}3098.7(\mathrm{OH}), 3050.4(\mathrm{Ar} \mathrm{C}- \\
\mathrm{H}), 2915.1\left(\mathrm{OCH}_{2}\right), 2360.5 \\
\left(\mathrm{CH}_{2}\right), 1735.0(\mathrm{C}=\mathrm{O}), 1596.0 \\
(\mathrm{C}=\mathrm{N}), 1492.0\left(\mathrm{NO}_{2}\right), 812.7 \\
(\mathrm{C}-\mathrm{Cl})\end{array}$ & $\begin{array}{l}14.0(\mathrm{br}, 1 \mathrm{H}, \mathrm{OH}), 10.8(\mathrm{~s}, 1 \mathrm{H} \\
\mathrm{CH}=\mathrm{N}), 8.7-6.9(\mathrm{~m}, 7 \mathrm{H}, \mathrm{Ar}-\mathrm{H}) \\
5.2\left(\mathrm{~s}, 2 \mathrm{H}, \mathrm{OCH}_{2}\right), 2.1(\mathrm{~s}, 2 \mathrm{H} \\
\left.\mathrm{CH}_{2}\right)\end{array}$ \\
\hline $3 \mathbf{h}$ & $2,4-\mathrm{Cl}$ & $\begin{array}{l}3-\mathrm{OCH}_{3}, \\
4-\mathrm{OH}\end{array}$ & $\begin{array}{l}3320 \text { and } 3200.2(2-\mathrm{OH}), \\
2900.2(\mathrm{Ar} \mathrm{C}-\mathrm{H}), 2923.6 \\
\left(\mathrm{OCH}_{2}\right), 2362.3\left(\mathrm{CH}_{2}\right) \\
1719.7(\mathrm{C}=\mathrm{O}), 1598.1 \\
(\mathrm{C}=\mathrm{N}), 754.3(\mathrm{C}-\mathrm{Cl})\end{array}$ & $\begin{array}{l}13.9(\mathrm{~s}, 1 \mathrm{H}, \mathrm{OH} \text { of } \mathrm{COOH}), 13.8 \\
\text { (br, } 1 \mathrm{H}, \mathrm{OH} \text { of benzylidine), } 9.8 \\
(\mathrm{~s}, 1 \mathrm{H}, \mathrm{N}=\mathrm{CH}), 7.8-6.9(\mathrm{~m}, 8 \mathrm{H}, \\
\mathrm{Ar}-\mathrm{H}), 5.2\left(\mathrm{~d}, 2 \mathrm{H}, \mathrm{OCH}_{2}\right), 3.9 \\
\left(\mathrm{~d}, 3 \mathrm{H}, \mathrm{OCH}_{3}\right), 2.1\left(\mathrm{~s}, 2 \mathrm{H}, \mathrm{CH}_{2}\right)\end{array}$ \\
\hline $3 \mathbf{i}$ & $2,4-\mathrm{Cl}$ & 4- & $3094.3(\mathrm{OH}), 3040.3(\mathrm{Ar} \mathrm{C}-$ & $13.8(\mathrm{br}, 1 \mathrm{H}, \mathrm{OH}), 9.6(\mathrm{~s}, 1 \mathrm{H}$ \\
\hline
\end{tabular}




\begin{tabular}{|c|c|c|c|c|}
\hline & & $\mathrm{N}\left(\mathrm{CH}_{3}\right)_{2}$ & $\begin{array}{l}\mathrm{H}), 2914.4\left(\mathrm{OCH}_{2}\right), 1730.4 \\
(\mathrm{C}=\mathrm{O}), 1586.2(\mathrm{C}=\mathrm{N}), 810.4 \\
(\mathrm{C}-\mathrm{Cl})\end{array}$ & $\begin{array}{l}\mathrm{N}=\mathrm{CH}), 7.6-6.6(\mathrm{~m}, 7 \mathrm{H}, \mathrm{Ar}-\mathrm{H}), \\
5.2\left(\mathrm{~d}, 2 \mathrm{H}, \mathrm{OCH}_{2}\right), 3.4(\mathrm{br}, 3 \mathrm{H}, \\
\left.\mathrm{CH}_{3}\right) 2.1\left(\mathrm{br}, 2 \mathrm{H}, \mathrm{CH}_{2}\right)\end{array}$ \\
\hline $3 \mathbf{j}$ & $2,4-\mathrm{Cl}$ & 4-OH & $\begin{array}{l}3108.0(\mathrm{OH}), 3047.0(\mathrm{Ar} \mathrm{C}- \\
\mathrm{H}), 2918.8\left(\mathrm{OCH}_{2}\right), 2753.0 \\
\left(\mathrm{CH}_{2}\right), 1720.3(\mathrm{C}=\mathrm{O}), 1610.3 \\
(\mathrm{C}=\mathrm{N}), 872.4(\mathrm{C}-\mathrm{Cl})\end{array}$ & $\begin{array}{l}13.9(\mathrm{t}, 1 \mathrm{H}, \mathrm{OH} \text { of Carboxylic } \\
\text { acid }), 11.6(\mathrm{~s}, 1 \mathrm{H}, \mathrm{OH} \text { of } \\
\text { benzylidine }), 10.0(\mathrm{~s}, 1 \mathrm{H}, \\
\mathrm{N}=\mathrm{CH}), 7.7-6.8(\mathrm{~m}, 7 \mathrm{H}, \mathrm{Ar}-\mathrm{H}), \\
5.2\left(\mathrm{~s}, 2 \mathrm{H}, \mathrm{OCH}_{2}\right), 2.8(\mathrm{~s}, 2 \mathrm{H}, \\
\left.\mathrm{CH}_{2}\right)\end{array}$ \\
\hline
\end{tabular}

Table 2. Physical and analytical data of compounds (2a-j) and (3a-j).

\begin{tabular}{|c|c|c|c|c|c|c|c|c|c|}
\hline \multirow{3}{*}{ Entry } & \multirow{3}{*}{$\mathbf{R}$} & \multirow{3}{*}{$\mathbf{R}_{1}$} & \multirow{3}{*}{$\begin{array}{l}\text { Yield } \\
(\%)\end{array}$} & \multirow{3}{*}{$\begin{array}{l}\text { Melting } \\
\text { point* } \\
\left({ }^{0} \mathrm{C}\right)\end{array}$} & \multirow{3}{*}{$\begin{array}{l}\text { Rf } \\
\text { Value\# }\end{array}$} & \multirow{3}{*}{$\begin{array}{l}\text { Molecular } \\
\text { formula }\end{array}$} & \multicolumn{3}{|c|}{ Analysis (\%) } \\
\hline & & & & & & & \multicolumn{3}{|c|}{ Found / (Calculated) } \\
\hline & & & & & & & $\mathbf{C}$ & $\mathbf{H}$ & $\mathbf{N}$ \\
\hline $2 \mathbf{a}$ & $\mathrm{H}$ & $\mathrm{H}$ & 70 & 142 & 0.52 & $\mathrm{C}_{16} \mathrm{H}_{14} \mathrm{~N}_{4} \mathrm{OS}$ & $\begin{array}{l}61.96 \\
(61.92)\end{array}$ & $\begin{array}{l}4.54 \\
(4.55)\end{array}$ & $\begin{array}{l}18.09 \\
(18.05)\end{array}$ \\
\hline $2 \mathbf{b}$ & $\mathrm{H}$ & $3-\mathrm{NO}_{2}$ & 68 & $184-86$ & 0.51 & $\mathrm{C}_{16} \mathrm{H}_{13} \mathrm{~N}_{5} \mathrm{O}_{3} \mathrm{~S}$ & $\begin{array}{l}54.04 \\
(54.08)\end{array}$ & $\begin{array}{l}3.62 \\
(3.69)\end{array}$ & $\begin{array}{l}19.73 \\
(19.71)\end{array}$ \\
\hline $2 c$ & $\mathrm{H}$ & $\begin{array}{l}3- \\
\mathrm{OCH}_{3}, \\
4-\mathrm{OH}\end{array}$ & 67 & 154 & 0.34 & $\mathrm{C}_{17} \mathrm{H}_{16} \mathrm{~N}_{4} \mathrm{O}_{3} \mathrm{~S}$ & $\begin{array}{l}57.26 \\
(57.29)\end{array}$ & $\begin{array}{l}4.56 \\
(4.52)\end{array}$ & $\begin{array}{l}15.77 \\
(15.72)\end{array}$ \\
\hline 2d & $\mathrm{H}$ & $\begin{array}{l}4- \\
\mathrm{N}\left(\mathrm{CH}_{3}\right)_{2}\end{array}$ & 66 & 164 & 0.50 & $\mathrm{C}_{18} \mathrm{H}_{19} \mathrm{~N}_{5} \mathrm{OS}$ & $\begin{array}{l}61.15 \\
(61.17)\end{array}$ & $\begin{array}{l}5.43 \\
(5.42)\end{array}$ & $\begin{array}{l}19.82 \\
(19.81)\end{array}$ \\
\hline $2 e$ & $\mathrm{H}$ & $4-\mathrm{OH}$ & 70 & $167-69$ & 0.39 & $\mathrm{C}_{16} \mathrm{H}_{14} \mathrm{~N}_{4} \mathrm{O}_{2} \mathrm{~S}$ & $\begin{array}{l}58.91 \\
(58.88)\end{array}$ & $\begin{array}{l}4.31 \\
(4.32)\end{array}$ & $\begin{array}{l}17.16 \\
(17.17)\end{array}$ \\
\hline $2 f$ & $\begin{array}{l}2,4- \\
\mathrm{Cl}\end{array}$ & $\mathrm{H}$ & 68 & $145-47$ & 0.43 & $\mathrm{C}_{16} \mathrm{H}_{12} \mathrm{Cl}_{2} \mathrm{~N}_{4} \mathrm{OS}$ & $\begin{array}{l}50.64 \\
(50.67)\end{array}$ & $\begin{array}{l}3.17 \\
(3.19)\end{array}$ & $\begin{array}{l}14.78 \\
(14.77)\end{array}$ \\
\hline $2 \mathrm{~g}$ & $\begin{array}{l}2,4- \\
\mathrm{Cl}\end{array}$ & $3-\mathrm{NO}_{2}$ & 70 & $212-15$ & 0.70 & $\mathrm{C}_{16} \mathrm{H}_{11} \mathrm{Cl}_{2} \mathrm{~N}_{5} \mathrm{O}_{3} \mathrm{~S}$ & $\begin{array}{l}45.32 \\
(45.30)\end{array}$ & $\begin{array}{l}2.59 \\
(2.61)\end{array}$ & $\begin{array}{l}16.49 \\
(16.51)\end{array}$ \\
\hline $2 \mathrm{~h}$ & $\begin{array}{l}2,4- \\
\mathrm{Cl}\end{array}$ & $\begin{array}{l}3- \\
\mathrm{OCH}_{3}, \\
4-\mathrm{OH}\end{array}$ & 72 & $205-07$ & 0.60 & $\mathrm{C}_{17} \mathrm{H}_{14} \mathrm{Cl}_{2} \mathrm{~N}_{4} \mathrm{O}_{3} \mathrm{~S}$ & $\begin{array}{l}48.04 \\
(48.01)\end{array}$ & $\begin{array}{l}3.33 \\
(3.32)\end{array}$ & $\begin{array}{l}13.18 \\
(13.17)\end{array}$ \\
\hline $2 \mathbf{i}$ & $\begin{array}{l}2,4- \\
\mathrm{Cl}\end{array}$ & $\begin{array}{l}4- \\
\mathrm{N}\left(\mathrm{CH}_{3}\right)_{2}\end{array}$ & 63 & $185-87$ & 0.50 & $\mathrm{C}_{18} \mathrm{H}_{17} \mathrm{Cl}_{2} \mathrm{~N}_{5} \mathrm{OS}$ & $\begin{array}{l}51.16 \\
(51.19)\end{array}$ & $\begin{array}{l}4.08 \\
(4.06)\end{array}$ & $\begin{array}{l}16.56 \\
(16.58)\end{array}$ \\
\hline $2 \mathbf{j}$ & $\begin{array}{l}2,4- \\
\mathrm{Cl}\end{array}$ & $4-\mathrm{OH}$ & 69 & $166-68$ & 0.50 & $\mathrm{C}_{16} \mathrm{H}_{12} \mathrm{Cl}_{2} \mathrm{~N}_{4} \mathrm{OS}$ & $\begin{array}{l}48.66 \\
(48.62)\end{array}$ & $\begin{array}{l}3.04 \\
(3.06)\end{array}$ & $\begin{array}{l}14.20 \\
(14.17)\end{array}$ \\
\hline $3 \mathbf{a}$ & $\mathrm{H}$ & $\mathrm{H}$ & 70 & 142 & 0.52 & $\mathrm{C}_{18} \mathrm{H}_{16} \mathrm{~N}_{4} \mathrm{O}_{3} \mathrm{~S}$ & $\begin{array}{l}58.66 \\
(58.68)\end{array}$ & $\begin{array}{l}4.34 \\
(4.38)\end{array}$ & $\begin{array}{l}15.20 \\
(15.21)\end{array}$ \\
\hline $3 \mathbf{b}$ & $\mathrm{H}$ & $3-\mathrm{NO}_{2}$ & 68 & $184-86$ & 0.51 & $\mathrm{C}_{18} \mathrm{H}_{15} \mathrm{~N}_{5} \mathrm{O}_{5} \mathrm{~S}$ & $\begin{array}{l}52.28 \\
(52.30)\end{array}$ & $\begin{array}{l}3.65 \\
(3.66)\end{array}$ & $\begin{array}{l}16.93 \\
(16.94)\end{array}$ \\
\hline $3 c$ & $\mathrm{H}$ & $\begin{array}{l}3- \\
\mathrm{OCH}_{3}, \\
4-\mathrm{OH}\end{array}$ & 67 & 154 & 0.34 & $\mathrm{C}_{19} \mathrm{H}_{18} \mathrm{~N}_{4} \mathrm{O}_{5} \mathrm{~S}$ & $\begin{array}{l}55.09 \\
(55.06)\end{array}$ & $\begin{array}{l}4.39 \\
(4.38)\end{array}$ & $\begin{array}{l}13.50 \\
(13.52)\end{array}$ \\
\hline 3d & $\mathrm{H}$ & $\begin{array}{l}4- \\
\mathrm{N}\left(\mathrm{CH}_{3}\right)_{2}\end{array}$ & 66 & 164 & 0.50 & $\mathrm{C}_{20} \mathrm{H}_{21} \mathrm{~N}_{5} \mathrm{O}_{3} \mathrm{~S}$ & $\begin{array}{l}58.36 \\
(58.38)\end{array}$ & $\begin{array}{l}5.12 \\
(5.14)\end{array}$ & $\begin{array}{l}17.04 \\
(17.02)\end{array}$ \\
\hline $3 \mathbf{e}$ & $\mathrm{H}$ & $4-\mathrm{OH}$ & 70 & $167-69$ & 0.39 & $\mathrm{C}_{18} \mathrm{H}_{16} \mathrm{~N}_{4} \mathrm{O}_{4} \mathrm{~S}$ & $\begin{array}{l}56.23 \\
(56.24)\end{array}$ & $\begin{array}{l}4.21 \\
(4.20)\end{array}$ & $\begin{array}{l}14.56 \\
(14.57)\end{array}$ \\
\hline
\end{tabular}




\begin{tabular}{|c|c|c|c|c|c|c|c|c|c|}
\hline 3f & $\begin{array}{l}2,4- \\
\mathrm{Cl}\end{array}$ & $\mathrm{H}$ & 69 & $145-47$ & 0.40 & $\mathrm{C}_{18} \mathrm{H}_{14} \mathrm{Cl}_{2} \mathrm{~N}_{4} \mathrm{O}_{3} \mathrm{~S}$ & $\begin{array}{l}49.45 \\
(49.44)\end{array}$ & $\begin{array}{l}3.24 \\
(3.23)\end{array}$ & $\begin{array}{l}12.80 \\
(12.81)\end{array}$ \\
\hline $3 \mathrm{~g}$ & $\begin{array}{l}2,4- \\
\mathrm{Cl}\end{array}$ & $3-\mathrm{NO}_{2}$ & 66 & $140-42$ & 0.44 & $\mathrm{C}_{18} \mathrm{H}_{13} \mathrm{Cl}_{2} \mathrm{~N}_{4} \mathrm{O}_{5} \mathrm{~S}$ & $\begin{array}{l}44.85 \\
(44.83)\end{array}$ & $\begin{array}{l}2.74 \\
(2.72)\end{array}$ & $\begin{array}{l}14.53 \\
(14.52)\end{array}$ \\
\hline $3 \mathbf{h}$ & $\begin{array}{l}2,4- \\
\mathrm{Cl}\end{array}$ & $\begin{array}{l}3- \\
\mathrm{OCH}_{3}, \\
4-\mathrm{OH}\end{array}$ & 67 & 207-09 & 0.35 & $\mathrm{C}_{19} \mathrm{H}_{16} \mathrm{Cl}_{2} \mathrm{~N}_{4} \mathrm{O}_{5} \mathrm{~S}$ & $\begin{array}{l}47.24 \\
(47.22)\end{array}$ & $\begin{array}{l}3.35 \\
(3.34)\end{array}$ & $\begin{array}{l}11.57 \\
(11.59)\end{array}$ \\
\hline $3 \mathbf{i}$ & $\begin{array}{l}2,4- \\
\mathrm{Cl}\end{array}$ & $\begin{array}{l}4- \\
\mathrm{N}\left(\mathrm{CH}_{3}\right)_{2}\end{array}$ & 70 & $177-79$ & 0.37 & $\mathrm{C}_{20} \mathrm{H}_{19} \mathrm{Cl}_{2} \mathrm{~N}_{5} \mathrm{O}_{3} \mathrm{~S}$ & $\begin{array}{l}50.03 \\
(50.01)\end{array}$ & $\begin{array}{l}3.98 \\
(3.99)\end{array}$ & $\begin{array}{l}14.60 \\
(14.58)\end{array}$ \\
\hline $3 \mathbf{j}$ & $\begin{array}{l}2,4- \\
\mathrm{Cl}\end{array}$ & $4-\mathrm{OH}$ & 65 & $190-92$ & 0.45 & $\mathrm{C}_{18} \mathrm{H}_{14} \mathrm{Cl}_{2} \mathrm{~N}_{4} \mathrm{O}_{4} \mathrm{~S}$ & $\begin{array}{l}47.71 \\
(47.69)\end{array}$ & $\begin{array}{l}3.12 \\
(3.11)\end{array}$ & $\begin{array}{l}12.38 \\
(12.36)\end{array}$ \\
\hline
\end{tabular}

${ }^{*}$ Recrystallization with ethanol. ${ }^{\text {\# }}$ Stationary Phase: Silica gel G, Mobile phase: Chloroform :

Benzene (1:1), Iodine vapors as visualizing agent.

Table 3. In-vitro antimicrobial activity of compounds (3a-j).

\begin{tabular}{|c|c|c|c|c|c|c|c|c|c|c|}
\hline \multirow{2}{*}{ Entry } & \multirow{2}{*}{$\mathbf{R}$} & \multirow{2}{*}{$\mathbf{R}_{1}$} & \multicolumn{8}{|c|}{ MIC values $(\mu \mathrm{g} / \mathrm{ml})$} \\
\hline & & & $\mathrm{Sa}$ & Bs & $\mathrm{Pa}$ & $\mathrm{Ec}$ & $\mathrm{Ca}$ & Cs & An & Af \\
\hline $3 \mathbf{a}$ & $\mathrm{H}$ & $\mathrm{H}$ & 16 & 32 & 32 & 16 & 4 & 4 & 4 & 4 \\
\hline $3 \mathbf{b}$ & $\mathrm{H}$ & $3-\mathrm{NO}_{2}$ & 8 & 64 & 8 & 8 & 16 & 4 & 4 & 4 \\
\hline $3 c$ & $\mathrm{H}$ & $\begin{array}{l}3-\mathrm{OCH}_{3}, \\
4-\mathrm{OH}\end{array}$ & $>128$ & 64 & 8 & 8 & 2 & 8 & 4 & 16 \\
\hline 3d & $\mathrm{H}$ & $4-\mathrm{N}\left(\mathrm{CH}_{3}\right)_{2}$ & 32 & 32 & 16 & 32 & 8 & 8 & 8 & 4 \\
\hline $3 \mathbf{e}$ & $\mathrm{H}$ & $4-\mathrm{OH}$ & 16 & 32 & 16 & 32 & 4 & 4 & 4 & 2 \\
\hline $3 f$ & $2,4-\mathrm{Cl}$ & $\mathrm{H}$ & 16 & 32 & 32 & 16 & 2 & 2 & 1 & 0.25 \\
\hline $3 g$ & $2,4-\mathrm{Cl}$ & $3-\mathrm{NO}_{2}$ & 64 & 64 & 64 & 64 & 0.25 & 1 & 1 & 1 \\
\hline $3 \mathbf{h}$ & $2,4-\mathrm{Cl}$ & $\begin{array}{l}3-\mathrm{OCH}_{3}, \\
4-\mathrm{OH}\end{array}$ & 16 & 16 & 8 & 8 & 1 & 0.25 & 0.25 & 0.25 \\
\hline $3 \mathbf{i}$ & $2,4-\mathrm{Cl}$ & $4-\mathrm{N}\left(\mathrm{CH}_{3}\right)_{2}$ & 32 & 32 & 32 & 32 & 1 & 1 & 1 & 1 \\
\hline $3 \mathbf{j}$ & $2,4-\mathrm{Cl}$ & $4-\mathrm{OH}$ & 64 & 8 & 64 & 64 & 1 & 0.25 & 0.25 & 0.25 \\
\hline Ceftriaxone & - & 0.5 & 0.5 & 0.5 & 0.5 & 0.5 & - & - & - & - \\
\hline Fluconazole & - & - & - & - & - & - & 1 & 1 & 1 & 1 \\
\hline $\begin{array}{l}\text { Streptococcu } \\
\text { (Ec). Candido } \\
\text { Aspergillus fi }\end{array}$ & $\begin{array}{l}\text { aureus } \\
\text { albicat } \\
\text { igatus }\end{array}$ & $\begin{array}{l}\text { Bacillus s } \\
\text { a), Cryptoc } \\
\end{array}$ & ilis (1 & $P$ & 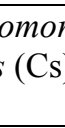 & 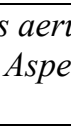 & os & $\begin{array}{l}\mathrm{Pa}), \mathrm{E} \\
\text { ger }\end{array}$ & $\begin{array}{l}\text { cherich } \\
\text { ), }\end{array}$ & a coli \\
\hline
\end{tabular}

\section{Conclusion}

A series of compounds 3a-j have been synthesised with satisfactory yield and screened for their antimicrobial activities. From the results, Compounds $\mathbf{3 h}, \mathbf{3} \mathbf{j}$, showed greater antifungal activity due to m-methoxy, $\mathrm{p}$-hydroxy groups and p-hydroxy group on imine phenyl ring against $A$. niger, $C$. neoformans and A. Fumigates, respectively. These can be regarded as strong candidates for future investigation. 


\section{References}

1. Olcay B, Hakan B, Molecules 2006, 11, 469-477.

2. Odd F C, J Antimicrob Chemother., 1993, 31, 463-471.

3. Parkinson T, Falconer D J, and Hitchcock C A, Antimicrob Agents Chemother., 1995, 39(8), 1696-1699.

4. Johnson E M, Warnock D W, Luker J, and Porter S R, Antimicrob Chemother., 1995, 35, 103-104.

5. Rex J H, Rinaldi M G and Pfallar M A, Antimicrob Agents Chemother., 1995, 39, 1-8.

6. Akbarzadeh T, Tabatabai S A, Khoshnoud M J, Shafaghi B and Shafiee A, Bioorg Med Chem., 2003, 11(5), 769-773.

7. Almasirad A, Mehrdad T, Kebriaeezadeh A, Mehrabi N, Dalvandia A, and Shafieea A, Bioorg Med Chem Lett., 2004, 14, 6057-6059.

8. Francis G, Cedric L, Fabrice P, Damien C, Patrice L, and Marc L, Bioorg Med Chem Lett., 2008, 18(6), 1820-1824.

9. Desai N C, Shukla H K and Thaker K A, J Indian Chem Soc., 1984, 6, 239-241.

10. Kucukguzel I, Esra T, Guniz Kucukguzel S, Rollas S, and De Clercq E, Eur J Med Chem., 2008, 43, 381-392.

11. Mahendra R S, Kiran K M, Hanimi R G, Tatikonda S, Chakravarthy A K, and Dolly P, Bioorg Med Chem., 2007, 15, 3997- 4008.

12. Upadhayaya R S, Sinha N, Jain S, Kishore N, Chandra R, and Arora S, Bioorg Med Chem., 2004, 12, 2225 - 2228.

13. Ronad P M, Hunashal R D, Satyanarayana D, and Maddi V S, Arzneim Forsch (Drug Res.)., 2008, 58, 641-646.

14. Ronad P M, Satyanarayana D, Hunashal R D, and Maddi V S, Arch. Pharm Chem Life Sci (Weinheim)., 2008, 341, 696-700.

15. Murry P R, Baron E J, Pfaller M A, Tenover F C, and Yolken R H, Am Soc Microbiol., 1995, 20, 556-563.

16. McFarland J, J Am Med Assoc., 1907, 14, 1176-1178. 


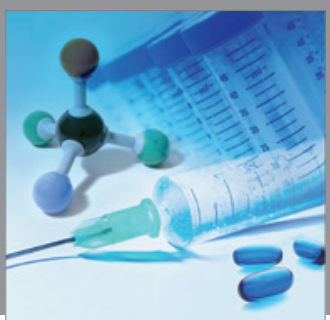

International Journal of

Medicinal Chemistry

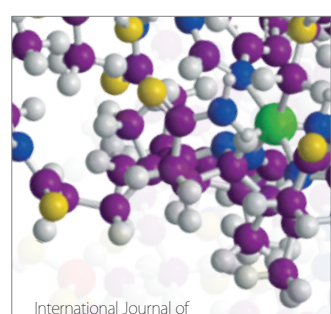

Carbohydrate Chemistry

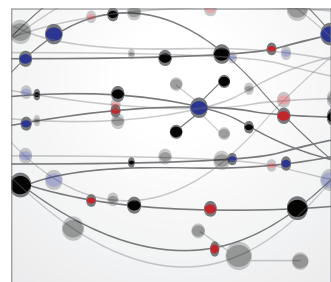

The Scientific World Journal
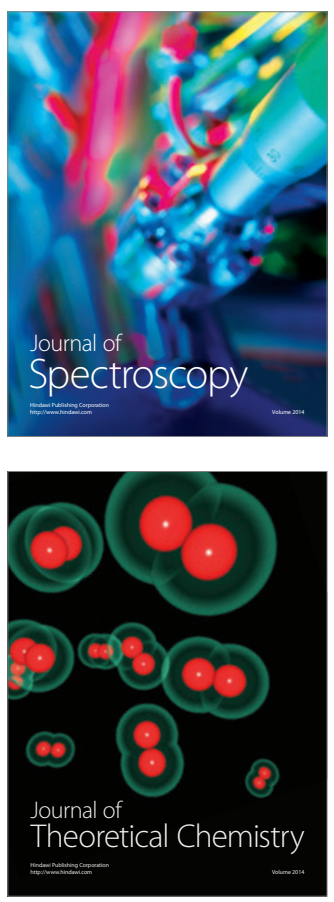
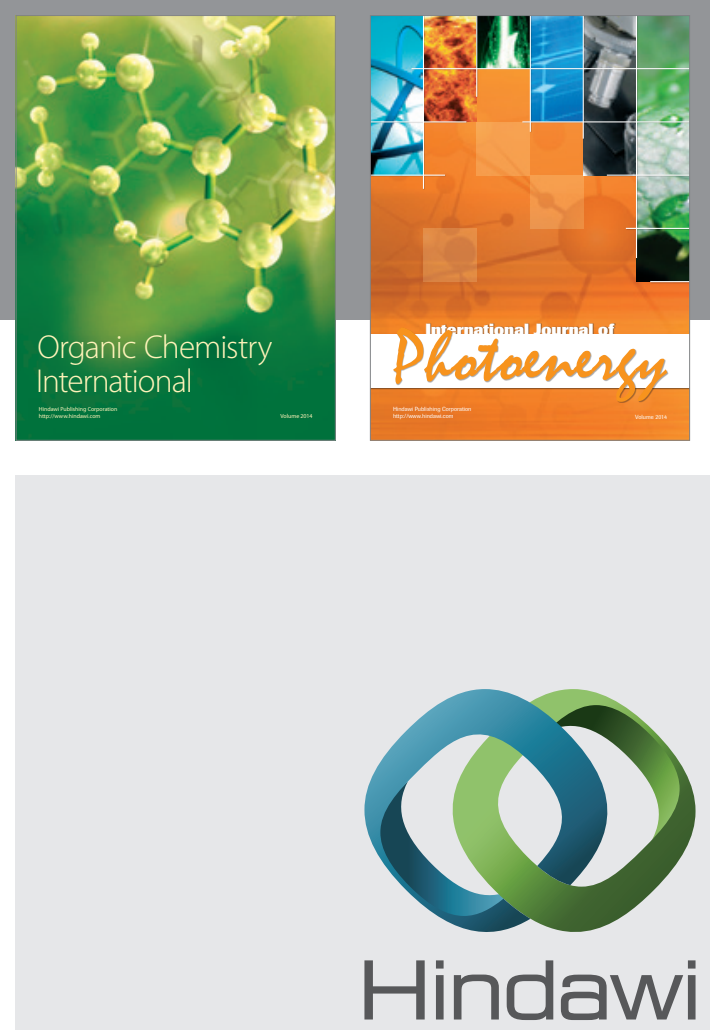

Submit your manuscripts at

http://www.hindawi.com
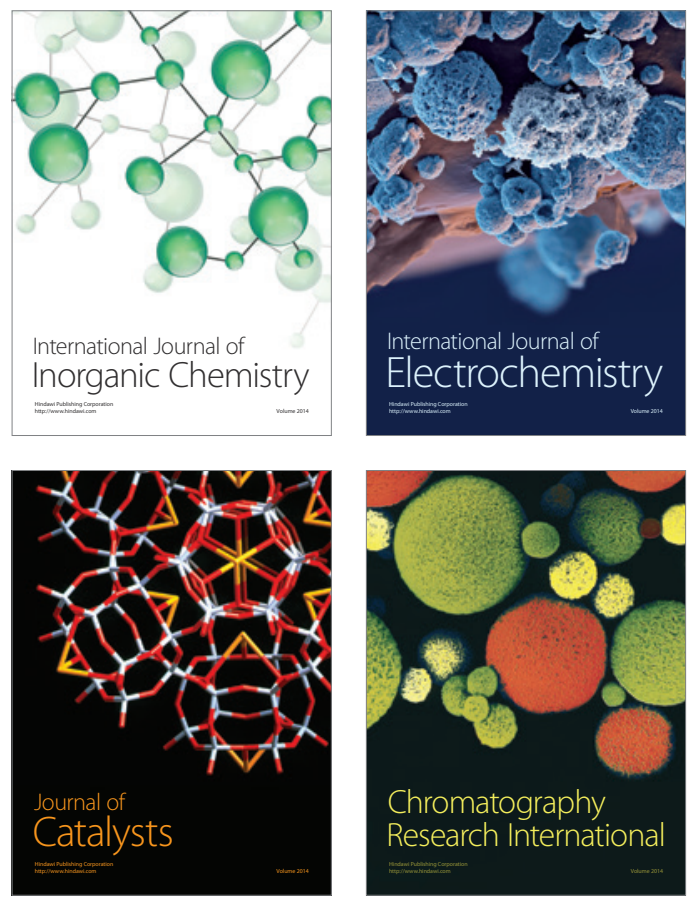
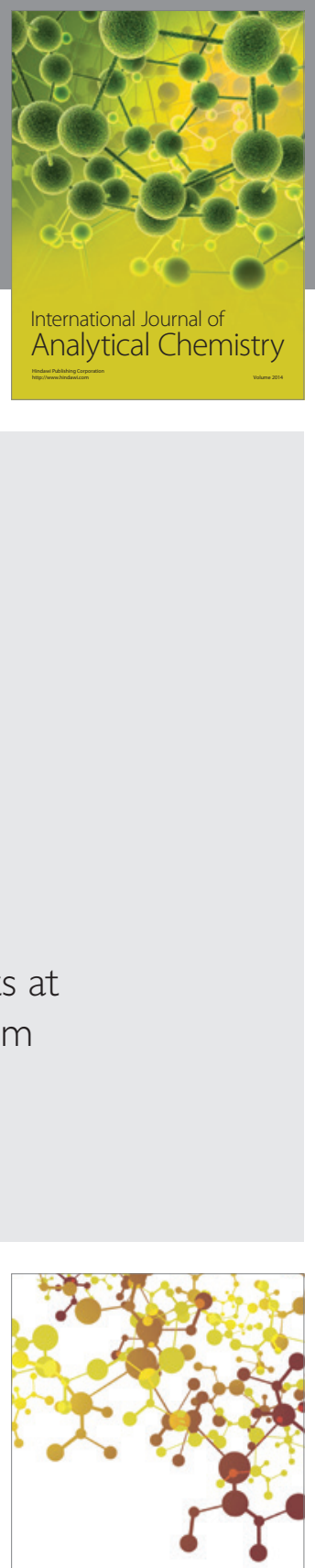

Journal of

Applied Chemistry
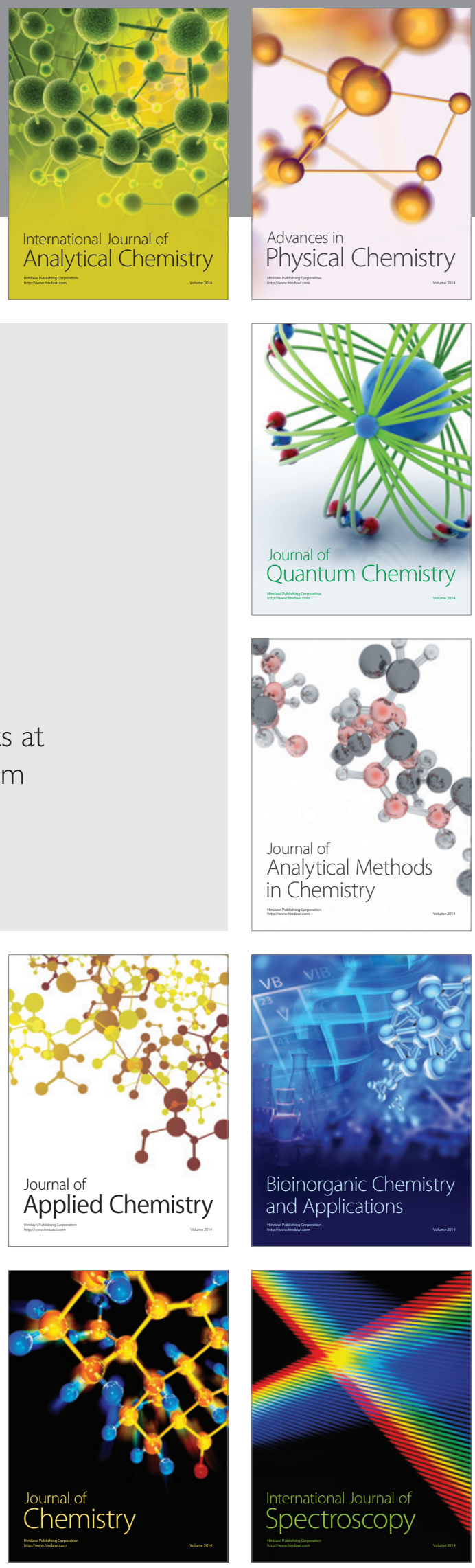